\title{
An Evaluation of the Early Pharmacodynamic Response After Simultaneous Initiation of Warfarin and Amiodarone
}

\author{
Stephanie B. Edwin, PharmD, Douglas L. Jennings, PharmD, and James S. Kalus, PharmD
}

Amiodarone inhibits the metabolism of warfarin. Previous studies characterizing this drug interaction have focused on the effect of adding amiodarone to stable doses of warfarin. The objective of this study was to assess whether simultaneous initiation of warfarin and amiodarone results in early alteration of the international normalized ratio (INR) response to warfarin. Patients initiated on warfarin and amiodarone during the same hospitalization were included in the amiodarone (AMIO) group. Patients initiated on warfarin alone $(n=42)$ were identified for the CONTROL group. The AMIO and CONTROL groups were matched based on age, gender, and ejection fraction $<40 \%$ using propensity score matching (final $n=18$ patients per group). Total and average daily warfarin dose was lower in the AMIO group, yet INR values were similar on each day between the 2 groups. More patients in the AMIO group had an INR greater than 2 during the 5-day observation period as compared to the CONTROL group. In addition, there were trends toward greater deviation from INR values expected with a 5-mg daily warfarin dose among AMIO group patients. Simultaneous initiation of warfarin and amiodarone leads to an enhanced pharmacodynamic response to warfarin early in therapy. Although these data should be viewed as hypothesis generating, cautious dosing and monitoring with simultaneous initiation of warfarin and amiodarone may be warranted.

Keywords: warfarin; amiodarone; anticoagulation; atrial fibrillation; drug interaction

Journal of Clinical Pharmacology, 2010;50:693-698

(C) 2010 The Author(s)

\begin{abstract}
$\mathrm{A}^{\mathrm{t}}$ trial fibrillation is the most common cardiac arrhythmia seen in clinical practice, affecting more than 2 million Americans. ${ }^{1}$ Thromboembolic complications, specifically embolic stroke, is one of the most significant contributors to morbidity, mortality, and cost associated with atrial fibrillation. The rate of stroke among patients with atrial fibrillation averages $5 \%$ per year, which is 2 to 7 times the rate for people without the disease. ${ }^{2}$ To reduce the risk of embolic stroke, lifelong anticoagulation is recommended with either warfarin or aspirin.

Although the use of antiarrhythmic agents has declined over the past few years, there is still a role for these agents in the management of selected

\footnotetext{
From the Department of Pharmacy, University of Michigan, Ann Arbor (Dr Edwin); and the Department of Pharmacy, Henry Ford Hospital, Detroit, Michigan (Dr Jennings, Dr Kalus). Submitted for publication June 24, 2009; revised version accepted September 8, 2009. Address for correspondence: James S. Kalus, Department of Pharmacy, Henry Ford Hospital, 2799 West Grand Boulevard, Detroit, MI 48202; e-mail: JKalus1@hfhs.org.

DOI: $10.1177 / 0091270009351885$
}

patients with atrial fibrillation. Amiodarone, a class III antiarrhythmic drug, is often selected based on known safety in patients with reduced left ventricular function and relatively high level of effectiveness in preserving normal sinus rhythm. ${ }^{3-5}$

Unfortunately, amiodarone often complicates the use of warfarin in patients with atrial fibrillation. Amiodarone potentiates the pharmacodynamic response of warfarin by inhibiting metabolism through CYP 2C9. The combination of warfarin and amiodarone is considered a clinically significant drug interaction that can lead to prolongation of the international normalized ratio (INR) and potentially increased risk of hemorrhagic complications. The literature surrounding this drug interaction has focused primarily on initiation of amiodarone in patients on stable doses of warfarin..$^{6-10}$ Based on this literature, empiric warfarin dose adjustment has been recommended when amiodarone is initiated in patients on stable doses of warfarin.

In clinical practice, amiodarone and warfarin are often initiated concurrently in patients with 
new-onset atrial fibrillation. Due to the unique pharmacokinetics and pharmacodynamics of both amiodarone and warfarin, it is difficult to predict the onset of this significant drug interaction. In fact, because it can take several weeks or months to achieve steady state with amiodarone, it could be hypothesized that the onset of the amiodarone-warfarin drug interaction could take several weeks or months as well. Therefore, it is unclear whether empiric warfarin dosage adjustment is necessary when warfarin and amiodarone are initiated concomitantly or whether the dose of warfarin should be reduced later in therapy. As such, management of this interaction is variable in clinical practice, and no standard of care exists.

In this study, we sought to better characterize the pharmacodynamic response to warfarin early in therapy when warfarin and amiodarone are initiated simultaneously.

\section{METHODS}

This study protocol was approved by the institutional review board at Henry Ford Hospital, a 903bed urban teaching hospital located in Detroit, Michigan. Medical records from patients concomitantly initiated on amiodarone and warfarin were retrospectively reviewed for patients admitted between January 1, 2005, and December 31, 2007. Patients who had orders for amiodarone loading doses of $\geq 600 \mathrm{mg} / \mathrm{d}$ on at least 4 of 5 days during the study period and warfarin processed on the same hospital day were evaluated for inclusion in the amiodarone (AMIO) group $(\mathrm{n}=18)$. Patients who were initiated on warfarin alone between September 2007 and April $2008(\mathrm{n}=42)$ were evaluated for inclusion in the CONTROL group. The different date ranges were used for the 2 study groups due to availability of easily retrievable electronic data. CONTROL patient data were accessed through an electronic pharmacy anticoagulation monitoring system that did not become available until September 2007. The anticoagulation monitoring system was deemed more appropriate for identifying the control population because it enabled simple identification of patients meeting inclusion and exclusion criteria without manual chart review. Given that patients eligible for inclusion in the AMIO group were fewer in number, a computerized system containing data prior to 2007 was required. Therefore, AMIO patient data were collected from an institutional medical record system, which reliably provided electronic data from January 1, 2005, to present. It should be noted that the primary source of data for both electronic systems used was the inpatient pharmacy computer system. The information contained in the systems is the same, but the ability to query data differs between the systems. Initial warfarin dose for all patients included in this analysis was $5 \mathrm{mg}$. Per hospital policy, warfarin doses were administered each day at 21:00.

Patients were excluded from both study groups if they had received vitamin $\mathrm{K}$ within the past 7 days, had underlying hepatic disease affecting coagulopathy (baseline INR $>1.40$ ), received warfarin prior to treatment with amiodarone, or were concomitantly using a drug known to have a highly probable interaction with warfarin. The following drugs were considered to have highly probable interactions with warfarin: ciprofloxacin, sulfamethoxazole, cotrimoxazole, erythromycin, fluconazole, isoniazid, levofloxacin, metronidazole, moxifloxacin, miconazole, voriconazole, diltiazem, fenofibrate, propafenone, propranolol, sulfinpyrazole, piroxicam, phenylbutazole, citalopram, entacapone, sertraline, griseofulvin, nafcillin, ribavirin, cholestyramine, rifampin, mesalamine, barbiturates, argatroban, and carbamazepine. ${ }^{11}$

Patient demographic characteristics, medical history, and inpatient drug therapy were evaluated. Warfarin dose, amiodarone dose/route of administration, and INR values during the first 5 days of therapy were also recorded.

The CONTROL group $(\mathrm{n}=42)$ was matched to the AMIO group $(n=18)$ in a $1: 1$ ratio using nearest neighbor propensity score matching. The match was designed to yield 2 groups of patients (18 patients per group) who were matched based on age, gender, and ejection fraction $(\mathrm{EF})<40 \%$. Matching was carried out with the freely available $\mathrm{R}$ software and "Matchit" package (www.r-project.org). All comparisons were made between the matched groups.

Several comparisons were made between groups to characterize the onset of the amiodarone-warfarin drug interaction. Mean daily warfarin dose, mean daily INR, change in INR over the evaluation period, and percent of patients achieving an INR value $\geq 2$ during the observation period were all compared between groups.

As a surrogate measure of "unpredictable response" to warfarin, the percentage of patients exceeding the maximum INR value predicted by the Crowther nomogram for a daily warfarin dose of 5 mg was compared between groups (deviation from the nomogram). The Crowther nomogram is a dosing 
Table I Baseline Patient Characteristics

\begin{tabular}{|c|c|c|c|}
\hline Variable & AMIO Group ( $n=18)$ & CONTROL Group $(\mathrm{n}=18)$ & $P$ Value \\
\hline Age, $y$, mean \pm SD & $70.1 \pm 11.4$ & $68.2 \pm 11.4$ & .633 \\
\hline Male, \% & 72.2 & 44.4 & .091 \\
\hline Weight, kg, mean \pm SD & $88.7 \pm 16.2$ & $83.2 \pm 16.1$ & .323 \\
\hline Left ventricular ejection fraction, $\%$, mean \pm SD & $39.7 \pm 18.7$ & $53.5 \pm 5.9$ & .017 \\
\hline Systolic heart failure, \% & 44.4 & 5.6 & .007 \\
\hline Hypertension, \% & 94.4 & 77.8 & .148 \\
\hline Coronary artery disease, \% & 77.8 & 61.1 & .278 \\
\hline Valve disease, $\%^{\mathrm{a}}$ & 16.7 & 5.6 & .289 \\
\hline Baseline international normalized ratio, mean \pm SD & $1.22 \pm 0.12$ & $1.13 \pm 0.90$ & .006 \\
\hline Warfarin days, $\mathrm{n}$, mean $\pm \mathrm{SD}$ & $4.67 \pm 0.59$ & $4.89 \pm 0.32$ & .172 \\
\hline Total AMIO dose, mg, mean \pm SD & $4777.8 \pm 1432.2$ & - & - \\
\hline Daily AMIO dose, mg, mean \pm SD & $1040.7 \pm 362.3$ & - & - \\
\hline
\end{tabular}

a. Valve disease $=$ moderate/severe disease as visualized by echocardiogram.

tool used during the initiation of warfarin. ${ }^{12}$ Based on an initial 5-mg dose of warfarin, this nomogram helps characterize a patient's pharmacodynamic response and provides guidance for dosage adjustments. Deviation from the nomogram was defined as an INR greater than 1.5 on day 2 of therapy, an INR greater than 1.9 on day 3 of therapy, and an INR greater than 3 on day 4 or 5 of therapy. These INR limits were defined as a "deviation" because exceeding these INR values suggests that the warfarin dose should be reduced to less than $5 \mathrm{mg}$. Rate of deviation from the nomogram was compared between groups at any time during the observation period, as well as on each individual day of the observation period.

\section{Statistical Analysis}

Continuous data are presented as mean \pm standard deviation, and categorical data are presented as percent. Continuous data were compared between the AMIO and CONTROL groups with a Student $t$ test. Fischer's exact test was used to compare categorical variables between groups. A log rank test was used to compare mean time to deviation from the Crowther nomogram.

\section{RESULTS}

\section{Demographics}

After propensity score matching, the AMIO and CONTROL groups were mostly balanced with regard to baseline characteristics (Table I). Left ventricular ejection fraction (LVEF) was lower in the AMIO group $(P=.017)$. This was due to a greater proportion of AMIO group patients having systolic heart failure $(P=.007)$. Baseline INR was also slightly different between groups $(P=.006)$. All patients in the AMIO group were on anticoagulation secondary to atrial fibrillation. The indication for anticoagulation varied in the CONTROL group; however, most patients were being treated for venous thromboembolism (88.9\%). Patients in the AMIO group received approximately $1 \mathrm{~g}$ of amiodarone daily (Table I).

\section{Warfarin Dose-Response Relationship}

Number of days of warfarin observed in the study was similar between the AMIO $(4.67 \pm 0.59$ days $)$ and CONTROL $(4.89 \pm 0.32)$ groups $(P=.172)$. Total warfarin dose over the evaluation period $(27.6 \pm 8.4$ $\mathrm{mg}$ vs $22.6 \pm 5.0 \mathrm{mg} ; P=.037)$ and average daily warfarin dose $(5.7 \pm 1.7 \mathrm{mg}$ vs $4.9 \pm 0.91 \mathrm{mg} ; P=$ .084) were lower in the AMIO group.

Despite the administration of lower warfarin doses to patients in the AMIO group, INR values were slightly but nonsignificantly higher in the AMIO group on days 2 to 4 after initiation of warfarin. Both groups experienced a similar change in INR between days 1 and 5 (AMIO: $+0.86 \pm 0.76$, CONTROL: +0.90 $\pm 0.90 ; P=.90)$. More patients in the AMIO group achieved an INR greater than 2 on each individual day of the evaluation period (Table II).

There was a trend for deviation from the Crowther nomogram to occur more frequently in the AMIO group (39\%) than in the CONTROL group $(17 \% ; P=$ .264). Deviation was uncommon on days 2 to 4 among the control patients, whereas deviation was more likely to occur on days 2 and 3 in the AMIO 
Table II International Normalized Ratio (INR) Values: AMIO Group vs CONTROL Group

\begin{tabular}{|c|c|c|c|c|c|}
\hline & Day & Day $2^{a}$ & Day 3 & Day 4 & Day 5 \\
\hline \multirow[t]{4}{*}{ AMIO } & Less than 2, \% & 100.0 & 66.7 & 44.4 & 33.3 \\
\hline & $2-3, \%$ & 0.0 & 22.2 & 50.0 & 61.1 \\
\hline & Greater than 3, \% & 0.0 & 11.1 & 5.6 & 5.6 \\
\hline & Any INR greater than $2, \%$ & 0.0 & 33.3 & 55.6 & 66.7 \\
\hline \multirow[t]{5}{*}{ CONTROL } & Less than $2, \%$ & 94.4 & 94.4 & 83.3 & 66.7 \\
\hline & $2-3, \%$ & 0.0 & 0.00 & 11.1 & 16.7 \\
\hline & Greater than 3, \% & 5.6 & 5.6 & 5.6 & 16.7 \\
\hline & Any INR greater than $2, \%$ & 5.6 & 5.6 & 16.7 & 33.3 \\
\hline & $P$ value $^{\mathrm{b}}$ & 1.00 & .088 & .035 & .094 \\
\hline
\end{tabular}

a. A total of 36 INR values were evaluated on each day except day 2. Only 34 INR values were available on this day.

b. Comparison of INR value greater than 2 between AMIO and CONTROL group.

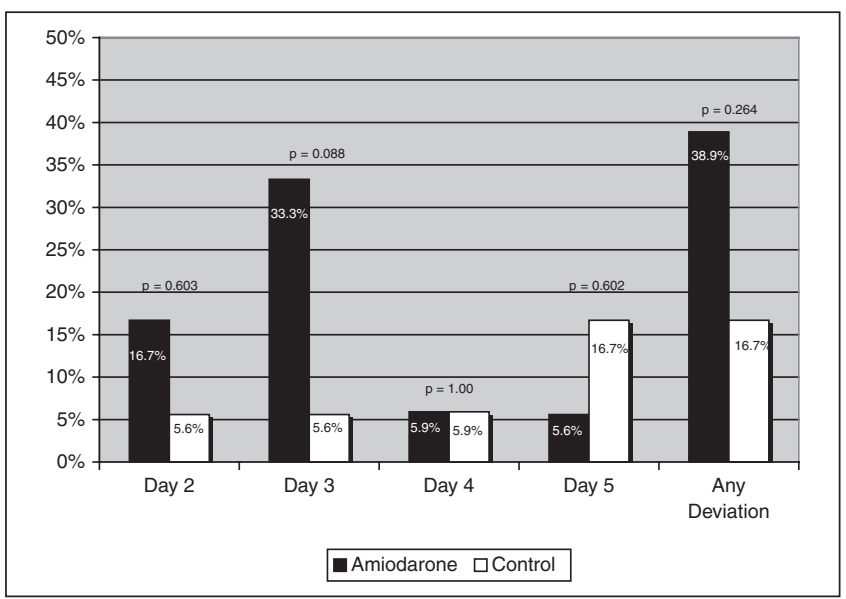

Figure 1. Deviation from the Crowther nomogram.

group (Figure 1). There was a trend for higher daily doses of amiodarone among those who deviated from the Crowther nomogram $(15.6 \pm 6.6 \mathrm{mg} / \mathrm{kg})$, as compared with those who did not deviate $(11.0 \pm 3.5 \mathrm{mg} /$ $\mathrm{kg} ; P=.077)$. There was also a trend toward a shorter mean time to deviation from the Crowther nomogram among AMIO patients $(P=.086$; Figure 2).

A post hoc analysis in which the 2 groups were analyzed in strata based on the presence or absence of systolic heart failure was also completed. In this analysis, similar trends were noted for any deviation from the Crowther nomogram among both patients with (AMIO: $37.5 \%$, CONTROL: $0 \% ; P=1.00$ ) and without (AMIO: $40.0 \%$, CONTROL: $17.6 \% ; P=.365$ ) systolic heart failure. Similar to the overall population, in the strata of patients without systolic heart failure, there were significantly more deviations from the Crowther nomogram on day 3 of therapy in the AMIO group $(40.0 \%$ vs $5.9 \% ; P=.047)$.

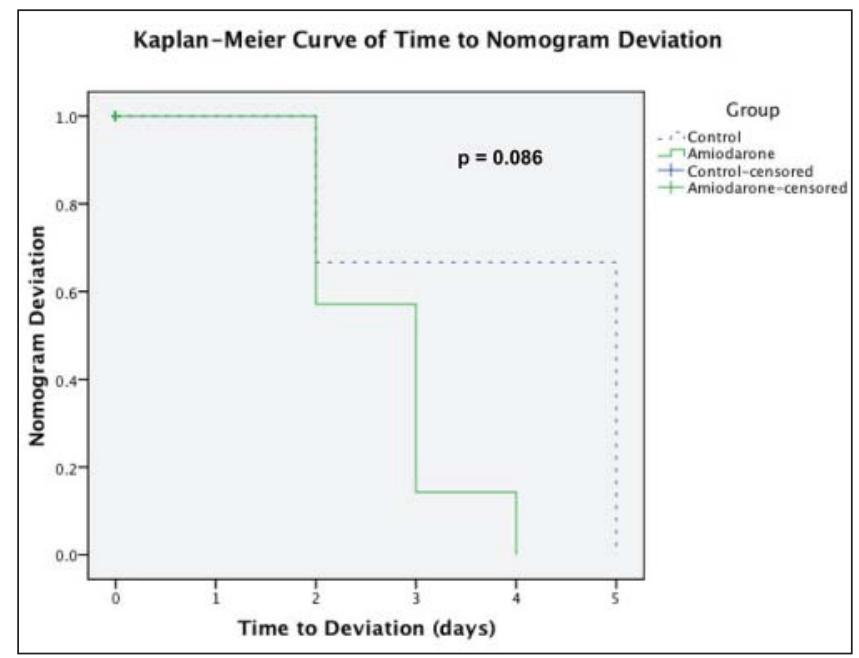

Figure 2. Time to nomogram deviation. Censored data reflect end of observation without any deviation from the nomogram.

\section{DISCUSSION}

In this study, differences in pharmacodynamic response were noted within the first few days of therapy between patients initiated on warfarin alone or warfarin plus amiodarone. Despite lower warfarin doses in the AMIO group, patients taking amiodarone achieved an INR greater than 2, more frequently. In addition, there were trends for deviation from the maximum INR value predicted by the Crowther nomogram to occur more frequently in the AMIO group. Although none of these measures independently proves early onset of the warfarin-amiodarone drug interaction, collectively these findings suggest that there is at least some inhibition of warfarin metabolism by amiodarone within the first few days of therapy. 
Previous literature evaluating the warfarin-amiodarone drug interaction has focused on initiation of amiodarone in patients on a stable dose of warfarin. ${ }^{6-8,13}$ As such, much of the focus of these reports has been on delayed alterations in warfarin pharmacodynamics as a result of amiodarone initiation. Therefore, a major difference between previous literature and this study is that this study evaluates the impact of amiodarone on warfarin pharmacodynamics in the first 5 days of initiation of both therapies.

A notable comparison between previous literature and the current study is the need for reduction in warfarin dosage. In the current study, warfarin dose was $18.1 \%$ lower in the first 5 days of concomitant warfarin and amiodarone administration. Warfarin dosage reduction ranged from $17.3 \%$ after 1 week of concomitant therapy in 1 study $^{6}$ to approximately $30 \%$ after 4 to 8 weeks of amiodarone in addition to warfarin therapy. ${ }^{7,8}$ Our data may suggest that the onset of the warfarin-amiodarone drug interaction is similar, regardless of whether amiodarone is added to stable warfarin dosage or if the 2 drugs are initiated at the same time. These data also suggest that the early impact of amiodarone on warfarin pharmacodynamics does not represent the full impact of this drug interaction that will manifest after weeks of therapy.

The pharmacodynamic response of patients in this study was compared to the response that would be expected when using the Crowther nomogram. The Crowther nomogram is a dosing tool that was originally described several years ago. ${ }^{12,14,15}$ The original reports relating to this dosing tool demonstrated that the use of 10-mg "loading" doses of warfarin in the first few days of warfarin therapy results in less efficient achievement of target INR ranges when compared to the use of a 5-mg loading dose. These studies also suggested that administration of 10-mg loading doses results in earlier achievement of an INR greater than 2 but also greater risk of developing a supratherapeutic INR (greater than 3). Of note, use of a 10-mg loading dose affected factor II levels similarly to the use of a 5-mg loading dose. However, factor VII and protein C levels were more dramatically lowered in the first few days of therapy with the 10-mg loading dose.

Comparisons of the current study data to these early reports yield interesting results. First, the 5-day pattern of more rapid change in INR is similar between the 10-mg group from previous literature $\mathrm{e}^{14,15}$ and the AMIO group in the current study. In addition, the AMIO group in the current study achieved an INR greater than 2 more frequently than the CONTROL group over a 5-day period. This is also similar to observations with the 10-mg group in previously published studies. ${ }^{14,15}$ Thus, these findings are consistent with the theory that inhibition of warfarin metabolism by amiodarone begins early after concomitant therapy has begun and suggest that concomitant initiation of amiodarone with warfarin may simulate initiation with higher doses of warfarin. Although this study did not evaluate protein C, factor II, or factor VII levels, one may hypothesize that similar results could have occurred in the setting of concomitant initiation of warfarin with amiodarone.

Some limitations of this study should be mentioned. First, despite the use of propensity score matching, the occurrence of systolic heart failure differed between the AMIO and CONTROL groups. This is notable because patients with systolic heart failure could have an altered pharmacodynamic response to warfarin. ${ }^{16}$ Therefore, the higher rate of heart failure in the AMIO group could explain the lower warfarin dose requirement and greater INR response. However, it seems that this difference did not influence our results because the post hoc analysis revealed similar influence of amiodarone on warfarin pharmacodynamics, regardless of heart failure status. Another potential limitation of these data is the short duration of the study. Although our intent was to identify early influence of amiodarone on warfarin pharmacodynamics, characterization of this drug interaction beyond 5 days would also be useful. Because previous literature has not addressed concomitant initiation of warfarin and amiodarone, as was evaluated in this study, the time course for the full impact of this drug interaction remains unclear. However, these data do suggest that at the very least, clinicians should anticipate the initial onset of this drug interaction in the first few days of therapy. It should also be noted that some of the analyses performed in this study were underpowered due to the small patient sample available. Finally, our study is limited by the use of a surrogate endpoint (INR) to measure the influence of the amiodarone-warfarin drug interaction. Although INR is well established as a measure of both efficacy and safety, it would be useful to know whether concomitant initiation of these 2 medications leads to altered clinical outcomes. This question is especially relevant in light of recent studies that demonstrate worsened clinical outcomes due to well-known pharmacokinetic/ pharmacodynamic drug interactions. ${ }^{17,18}$ However, this study could provide pilot data to justify work evaluating the impact of concomitant warfarin and amiodarone on clinical outcomes in the future. 


\section{CONCLUSION}

Simultaneous initiation of warfarin and amiodarone likely leads to early inhibition of warfarin metabolism and an enhanced pharmacodynamic response to warfarin early in therapy. Although these data should be viewed as hypothesis generating, cautious dosing and monitoring with simultaneous initiation of warfarin and amiodarone may be warranted.

This paper was presented as a poster, "Initial Clinical Observations When Simultaneously Initiating Warfarin and Amiodarone," at the American Society of Health System Pharmacists Midyear Clinical Meeting in Las Vegas, Nevada, on December 3, 2007.

\section{REFERENCES}

1. Go AS, Hylek EM, Phillips KA, et al. Prevalence of diagnosed atrial fibrillation in adults: national implications for rhythm management and stroke prevention: the Anticoagulation and Risk Factors in Atrial Fibrillation (ATRIA) Study. JAMA. 2001; 285:2370-2375.

2. Fuster V, Ryden LE, Asinger RW, et al. American College of Cardiology/American Heart Association Task Force on Practice Guidelines. European Society of Cardiology Committee for Practice Guidelines and Policy Conferences North American Society of Pacing and Electrophysiology. ACC/AHA/ESC Guidelines for the Management of Patients With Atrial Fibrillation: Executive Summary: A Report of the American College of Cardiology/American Heart Association Task Force on Practice Guidelines and the European Society of Cardiology Committee for Practice Guidelines and Policy Conferences. Developed in collaboration with the North American Society of Pacing and Electrophysiology. Circulation. 2001;104:2118-2150.

3. Roy D, Talajic M, Dorian P, et al. Amiodarone to prevent recurrence of atrial fibrillation. $N$ Engl J Med. 2000;342:913-920.

4. Singh BN, Singh SN, Reda DJ, et al. Amiodarone versus sotalol for atrial fibrillation. $N$ Engl J Med. 2005;352:1861-1872.

5. Lafuente-Lafuente $\mathrm{C}$, Mouly $\mathrm{S}$, Longas-Tejero MA, et al. Antiarrhythmics for maintaining sinus rhythm after cardioversion of atrial fibrillation. Cochrane Database Syst Rev. 2007;(4): CD005049.

6. Sanoski CA, Bauman JL. Clinical observations with the amiodarone/warfarin interaction: dosing relationships with long-term therapy. Chest. 2002;121:19-23.

7. Kerin NZ, Blevins RD, Goldman L, et al. The incidence, magnitude, and time course of the amiodarone-warfarin interaction. Arch Intern Med. 1988;148:1779-1781.

8. Almog S, Shafran N, Halkin H, et al. Mechanism of warfarin potentiation by amiodarone: dose- and concentration-dependent inhibition of warfarin elimination. Eur J Clin Pharmacol. 1985; 28:257-261.

9. Watt AH, Stephens MR, Buss DC, et al. Amiodarone reduces plasma warfarin clearance in man. $\mathrm{Br} J$ Clin Pharmacol. 1985;20:707-709.

10. Hamer A, Peter T, Mandel WJ, et al. The potentiation of warfarin anticoagulation by amiodarone. Circulation. 1982;65:1025-1029.

11. Holbrook AM, Pereira JA, Labris R, et al. Systematic overview of warfarin and its drug and food interactions. Arch Intern Med. 2005;165:1095-1106.

12. Crowther MA, Harrison L, Hirsh J. Warfarin: less may be better. Ann Intern Med. 1997;127:333.

13. Lu Y, Won KA, Nelson BJ, Qi D, Rausch DJ, Asinger RW. Characteristics of the amiodarone-warfarin interaction during long-term follow-up. Am J Health Syst Pharm. 2008;65:947-952.

14. Crowther MA, Ginsberg JB, Kearon C, et al. A randomized trial comparing 5-mg and 10-mg warfarin loading doses. Arch Intern Med. 1999;159:46-48.

15. Harrison L, Johnston M, Massicotte MP, Crowther M, Moffat K, Hirsh J. Comparison of 5-mg and 10-mg loading doses in initiation of warfarin therapy. Ann Intern Med. 1997;126:133-136.

16. Ansell J, Hirsh J, Hylek E, Jacobson A, Crowther M, Palareti G. Pharmacology and management of vitamin K antagonists. Chest. 2008;133:160S-198S.

17. Juurlink DN, Gomes T, Ko DT, et al. A population-based study of the drug interaction between proton pump inhibitors and clopidogrel. CMAJ. 2009;180:713-718.

18. Ho PM, Maddox TM, Wang L, et al. Risk of adverse outcomes associated with concomitant use of clopidogrel and proton pump inhibitors following acute coronary syndrome. JAMA. 2009;301: 937-944. 